\title{
IMPLEMENTATION OF ECG SIGNAL ACQUISITION AND TRANSMISSION THROUGH BLUETOOTH TECHNOLOGY
}

\author{
P.Vignesh ${ }^{1}$ \\ ${ }^{1} P G$ Scholar, Nandha Engineering College, Erode-52
}

\begin{abstract}
The proposed system of this paper introduces the ECG signal acquisition by using electrodes and transmits the obtained ECG signal through Bluetooth technology. To achieve this ECG signal acquisition and transmission, we have to use three nodes as ECG acquisition node, Wireless communication node and signal monitoring node. For transmission and receiving process, the Bluetooth from the ECG signal acquisition node will be act as the sender (server) and the Bluetooth from monitoring node will act as the receiver. This paper is useful for analyzing the obtained ECG signal and can be used for the purpose of telemedicine systems.
\end{abstract}

Keywords- ECG signals, WLAN, Bluetooth technology, Monitoring, Telemedicine Systems

\section{INTRODUCTION}

The telemedicine system is one which is the most emerging trend in day today life. This telemedicine system is emerging because of reducing the total cost of the patient especially for poor patients and the patients from border areas by reducing travelling allowances, accommodation charges as well as the amount to meet the specialists for some complicated diseases.

By the help of the telemedicine systems, the patients may not need to meet the specialists and the patients can get effective treatments by their own places or by the local hospitals. Because of these reasons, the people from villages are interested on telemedicine systems.

In this telemedicine systems, the patients are not needed to meet the specialists continuously until their problems gets cure. Some data acquisition nodes depends upon the disease of the patients will be placed with the patient's body and the acquired data will be transmitted to the nearest hospital or to the specialists by the communication nodes. These communication nodes may be a GSM technique or CDMA technique or otherwise may be wireless local area networks (WLAN) such as Bluetooth, ZIGBEE, Wi-Fi, etc., The transmitted signals from communication node will be received by the same technology in the receiver end and then this signal will be monitored in the monitor. This detailed process will be done in the telemedicine systems.

\section{OBJECTIVE}

The major objective of this paper is to acquire the ECG signal from a human body and transmitting that acquired ECG signal from one place to another place by using the wireless Bluetooth technology.

$* * *$

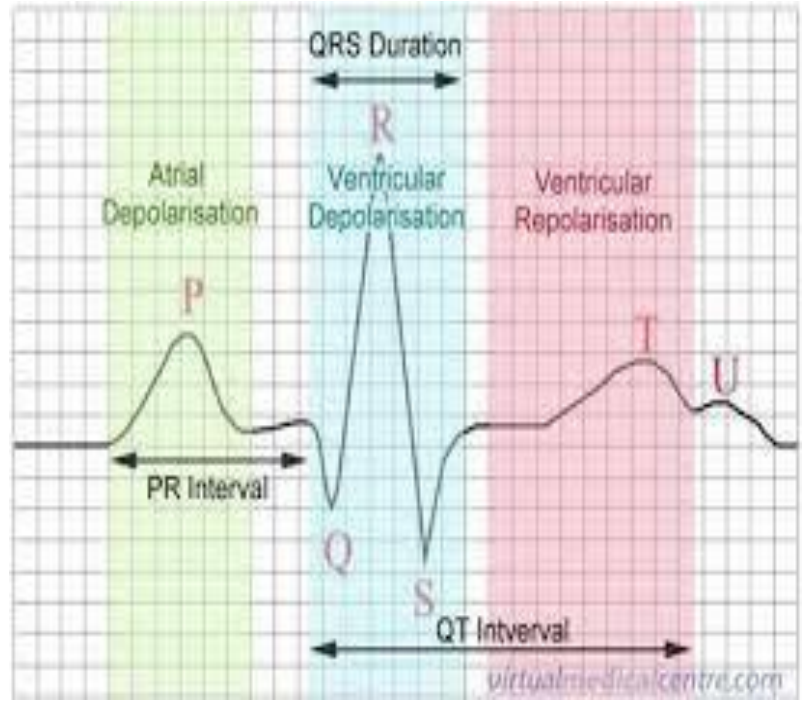

Fig 1 ECG wave time interval.

The above figure shows the time interval for each $\mathrm{P}, \mathrm{Q}, \mathrm{R}$, $\mathrm{S}, \mathrm{T}, \mathrm{U}$ intervals in an ECG wave form. This time interval is common to all normal human beings except the cardiac patients. The ECG acquisition node should be effectively clear to acquire the proper i.e., appropriate ECG signal from heart.

In this paper, the Bluetooth technology is used for transferring and receiving of signals. The Bluetooth will be act as a transmitter in human end and act as a receiver in another end.

\section{METHODOLOGY}

\subsection{Block Diagram}

The following is the block diagram for ECG signal acquisition and continuous transferring of that signal by the help of Bluetooth technology. 


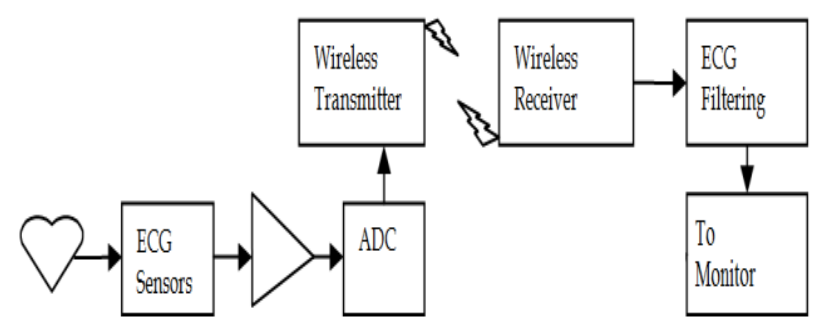

Fig 2 ECG Signal Transmission

\subsection{Block Description}

The ECG signal is acquired from human body with the help of ECG electrodes. There are three types of leads used to acquire the ECG signal from human body. We can use any one of these lead types to acquire the ECG signal.
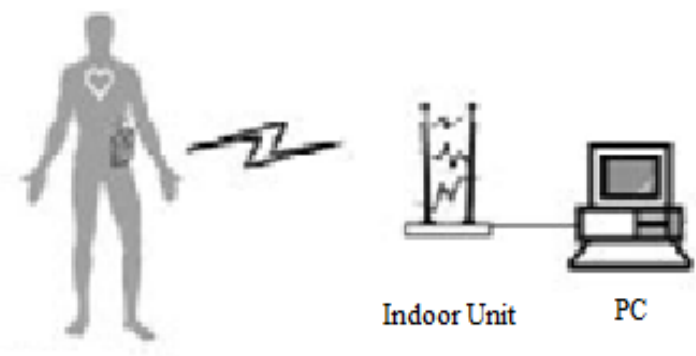

Indoor Unit

$\mathrm{PC}$

ECG acquisition

\& transmission

Fig 3 Detailed process.

In this above figure, the ECG signal is taken with the help of ECG sensors. These ECG sensors can be placed at the chest of the patient or otherwise at the wrist. The acquired ECG signals from the ECG sensors are in the form of analog signal. This analog signal is given to the ADC unit to convert it into digital signal. It is very difficult to transfer an analog signal through Bluetooth technology. The data loss will be very high and the data will be collapsed if we transmit as a analog signal. So, the analog signal is converted into digital signal.

After completing the signal conversion process, the converted digital signal is given to the Bluetooth transmitter unit. This Bluetooth transmitter is pre-paired with another Bluetooth receiver. This paired Bluetooth receiver will start receiving process when the Bluetooth transmitter starts to send the data.

Then the received signal is filtered for removing the unwanted signal with is occurred during data transmission. The ECG filtering unit is used to filter the received digital ECG signal. Then again the signal is converted into the necessary form to monitor the signal in a monitor. So, the total system is act as a real time system.

\section{FEATURES}

The important features of this proposed system are,

$>\quad$ The Bluetooth technology is mostly used in most electronic equipments like mobile phones, tablets, laptops, etc., So we can configure our personal mobile phones as the receiver end.

$>\quad$ We can save time by avoiding the waiting time to meet the specialists and by reducing the travelling to hospitals.

$>\quad$ We can also save money by avoiding accommodation at the hospitals.

$>\quad$ This system is very useful for the cardiac patients, who are not interested to stay at hospitals.

$>\quad$ This system also can be implemented even in remote (mobile) patients too.

$>\quad$ This system can be easily implemented in society.

These are some features of the proposed model.

\section{CONCLUSION}

This paper shows the novel approach of the wireless data transmission using Bluetooth technology for ECG signal monitoring. The important specification of this paper is based on the real time monitoring of biosignals. This above process can be also done by using LAN technology and by another wired data transmission technologies.

\section{REFERENCES}

[1] M. B. khodabakhshi, and A. Janqorbani, "Screening and ECG signal using a mobile phone, along with the diagnosis", Thesis, Shahed University, 2008.

Chan, Brady, Harrigan, ornato, and rosen, ECG in Emergency Medicine and Acute Care, 2005, PP.8082.

[3] T. K. Kho, Rosli Besar, Y. S. Tan, K. H. Tee, K. C. Ong, "Bluetooth-enabled ECG Monitoring System", Tencon 2005 IEEE Region 10, 2005, pp.1-5.

[4] R. Bouhenguel, I. Mahgoub, and M. Ilyas, "Bluetooth security in wearable computing applications," in Proceedings of the International Symposium on High Capacity Optical Networks and Enabling Technologies, pp. 182-186, November 2008.

[5] G. Clifford, A. Francisci, and P. E. McSharry, Advanced Methods and Tools for ECG Data Analysis, 2006, pp.68-73. 